\title{
NOTE ON THE FIRST CESARO MEAN OF THE DERIVED FOURIER SERIES
}

R. MOHANTY AND M. NANDA

1. Let $f(t)$ be integrable $L$ in $(-\pi, \pi)$ and periodic with period $2 \pi$ and let

$$
f(t) \sim \frac{1}{2} a_{0}+\sum_{1}^{\infty}\left(a_{n} \cos n t+b_{n} \sin n t\right)=\frac{1}{2} a_{0}+\sum_{1}^{\infty} A_{n}(t) .
$$

Then the differentiated series of $\sum_{1}^{\infty} A_{n}(t)$ is

$$
\sum_{1}^{\infty} n\left(b_{n} \cos n t-a_{n} \sin n t\right)=\sum_{1}^{\infty} n B_{n}(t) \text {. }
$$

We write

$$
\psi(t)=f(x+t)-f(x-t), \quad g(t)=\frac{f(x+t)-f(x-t)}{2 \sin (t / 2)} .
$$

Let $S_{n}(x)$ be the $n$th partial sum and $t_{n}(x)$ the first arithmetic mean of the series (1), where

$$
\begin{gathered}
S_{n}(x)=\sum_{r=1}^{n} r B_{r}(x), \\
t_{n}(x)=\left(S_{1}+S_{2}+\cdots+S_{n}\right) / n .
\end{gathered}
$$

The object of the present note is to prove

THEOREM 1. If 1

$$
\int_{0}^{t}|g(u)-c| d u=o(t) \quad \text { when } t \rightarrow 0
$$

then $t_{n}=o(\log n)$.

2. Proof of Theorem 1 . We have

(6)

$$
\begin{aligned}
r B_{r}(x) & =\frac{1}{\pi} \int_{0}^{\pi}\{f(x+t)-f(x-t)\} r \sin r t d t \\
& =\frac{1}{\pi} \int_{0}^{\pi} \psi(t) r \sin r t d t .
\end{aligned}
$$

Received by the editors October 26, 1953.

'Throughout the present note there is the tacit assumption that $g(t)$ is $L$ in $(0, \pi)$ and $c$ a function of $x$. 
Hence

$$
\begin{aligned}
S_{n}(x) & =\frac{1}{\pi} \int_{0}^{\pi} \psi(t) d t \sum_{r=1}^{n} r \sin r t \\
& =-\frac{1}{\pi} \int_{0}^{\pi} \psi(t) \frac{d}{d t}\left\{\frac{1}{2}+\sum_{1}^{n} \cos r t\right\} d t \\
& =-\frac{1}{\pi} \int_{0}^{\pi} \psi(t) \frac{d}{d t}\left\{\frac{\sin (n+1 / 2) t}{2 \sin (t / 2)}\right\} d t .
\end{aligned}
$$

Now from (4) and (7) we have

$$
\begin{aligned}
& t_{n}(x)=\frac{1}{n} \sum_{k=1}^{n} S_{k} \\
& =-\frac{1}{\pi n} \int_{0}^{\pi} \psi(t) \frac{d}{d t}\left\{\frac{1}{2 \sin (t / 2)} \sum_{k=1}^{n} \sin \left(k+\frac{1}{2}\right) t\right\} d t \\
& =-\frac{1}{\pi n} \int_{0}^{\pi} \psi(t) \frac{d}{d t}\left[\frac{\cos (t / 2)\{\cos (t / 2)-\cos (n+1 / 2) t\}}{4 \sin ^{2}(t / 2)}\right. \\
& \left.+\frac{1}{2}\left\{\frac{\sin (n+1 / 2) t}{2 \sin (t / 2)}-\frac{1}{2}\right\}\right] d t \\
& =-\frac{1}{\pi n} \int_{0}^{\pi} \psi(t) \frac{d}{d t}\left\{\frac{\cos t}{4 \sin ^{2}(t / 2)}-\frac{\cos (n+1) t}{4 \sin ^{2}(t / 2)}\right\} d t \\
& =\frac{1}{\pi n} \int_{0}^{\pi} g(t) \cos (t / 2) \frac{1-\cos (n+1) t}{2 \sin ^{2}(t / 2)} d t \\
& -\frac{n+1}{n} \frac{1}{\pi} \int_{0}^{\pi} g(t) \frac{\sin (n+1) t}{2 \sin (t / 2)} d t \\
& =\frac{1}{\pi n} \int_{0}^{\pi} g^{*}(t) \cos (t / 2)\left(\frac{\sin ^{2}((n+1) t / 2)}{\sin ^{2}(t / 2)}\right) d t \\
& -\frac{n+1}{n} \frac{1}{\pi} \int_{0}^{\pi} g^{*}(t) \frac{\sin (n+1) t}{2 \sin (t / 2)} d t \\
& +\frac{c}{\pi n} \int_{0}^{\pi} \cos (t / 2)\left(\frac{\sin ^{2}((n+1) t / 2)}{\sin ^{2}(t / 2)}\right) d t \\
& -\frac{c}{\pi} \frac{n+1}{n} \int_{0}^{\pi} \frac{\sin (n+1) t}{2 \sin (t / 2)} d t \quad\left(\text { where } g(t)-c=g^{*}(t)\right) \\
& =I_{1}+I_{2}+I_{3}+I_{4} \text {, say. }
\end{aligned}
$$


Now using the proof of the Fejer-Lebesgue theorem for summability $(c, 1)$ of the Fourier series ${ }^{2}$ with $g^{*}(t)$ in place of $\phi(t)$, it can be proved that

$$
I_{1}=o(1) .
$$

Using the argument similar to that employed in the proof of a wellknown result ${ }^{3}$ and the Condition (5) of Theorem 1, we have

$$
I_{2}=o(\log n) \text {. }
$$

Again

$$
\begin{aligned}
\left|I_{3}\right| & =\frac{|c|}{\pi n} \int_{0}^{\pi} \cos (t / 2) \frac{\sin ^{2}((n+1) t / 2)}{\sin ^{2}(t / 2)} d t \\
& <o(1)+\frac{|c|}{\pi n} \int_{0}^{\pi} \frac{\sin ^{2}((n+1) t / 2)}{t^{2}} d t \\
& =o(1)+\frac{|c|}{\pi} \frac{n+1}{n} \int_{0}^{(n+1) \pi / 2} \frac{\sin ^{2} \theta}{\theta^{2}} d \theta \\
& \rightarrow \frac{|c|}{\pi} \int_{0}^{\infty} \frac{\sin ^{2} \theta}{\theta^{2}} d \theta \\
& =|c| / 2 .
\end{aligned}
$$

Lastly

$$
\begin{aligned}
I_{4} & =-\frac{c}{\pi} \frac{n+1}{n} \int_{0}^{\pi} \frac{\sin (n+1) t}{\sin (t / 2)} d t \\
& =-\frac{c}{\pi} \frac{n+1}{n}\left[\int_{0}^{(n+1) \pi} \frac{\sin \theta}{\theta} d \theta+o(1)\right] \\
& \rightarrow-\frac{c}{\pi} \int_{0}^{\infty} \frac{\sin \theta}{\theta} d \theta \\
& =-c / 2 .
\end{aligned}
$$

Hence by (7), (9), (10), (11), and (12), we have

$$
t_{n}=o(\log n) \text {. }
$$

3. Using relation (13) above we prove

THEOREM 2. If

$$
g(t) \rightarrow c,
$$
when $t \rightarrow 0$

${ }^{2}$ The theory of functions, E. C. Titchmarsh, 1939, p. 415.

${ }^{3}$ Fourier series by G. H. Hardy and W. W. Rogosinski, Theorem 64, p. 49. 
then

$$
\sum_{r=1}^{\infty} \frac{r B_{r}(x)}{\log (r+1)}
$$

To prove Theorem 2 we require the following lemmas:

Lemma I. ${ }^{4}$ If $\sum_{r=1}^{\infty} U_{r}$ is summable $(c, k+1)$, where $k>-1$, then $a$ necessary and sufficient condition that it should be summable $(c, k)$ is that

$$
\sum_{r=1}^{n} A_{n-r}^{k} U_{r} \cdot r=\text { on } n^{k+1} ; \text { where } A_{m}^{k}=\frac{(k+1)(k+2) \cdots(k+m)}{m !} .
$$

Lemma II. If $\int_{0}^{t}\left|g^{*}(u)\right| d u=o(t)$, then $S_{n}(x)=o(n)$.

Proof of Lemma II.

$$
\begin{aligned}
S_{n}(x)= & -\frac{1}{\pi} \int_{0}^{\pi} \psi(t) \frac{d}{d t}\left\{\frac{\sin (n+1 / 2) t}{2 \sin (t / 2)}\right\} d t \\
= & \frac{1}{\pi} \int_{0}^{\pi} g(t) \frac{\cos (t / 2) \sin (n+1 / 2) t}{2 \sin (t / 2)} d t \\
& -\frac{1}{\pi}\left(n+\frac{1}{2}\right) \int_{0}^{\pi} g(t) \cos \left(n+\frac{1}{2}\right) t d t \\
= & o(\log n)+o(n)=o(n),
\end{aligned}
$$

using the condition of Lemma II.

Proof of Theorem 2. If $g(t) \rightarrow c$ when $t \rightarrow 0$, then $\sum_{r=1}^{\infty} r B_{r}(x)$ is summable $(c, 2) ; 5$ hence in view of the case $k=1$ of Lemma I above, it is enough for the proof of Theorem 2 to show that

$$
\sum_{r=1}^{n} A_{n-r}^{\prime} r B_{r}(x) \frac{r}{\log (r+1)}=o\left(n^{2}\right) .
$$

Applying Abel's transformation twice, we find

$$
\begin{aligned}
\sum_{r=1}^{n} A_{n-r}^{\prime} r B_{r}(x) \frac{r}{\log (r+1)}= & \sum_{r=1}^{n-2}(r+1) t_{r} \Delta^{2}\left(A_{n-r}^{\prime} \frac{r}{\log (r+1)}\right) \\
& +n t_{n-1} \Delta\left(\frac{n}{\log n}\right)+S_{n} \frac{n+1}{\log (n+1)} \\
& =T_{1}+T_{2}+T_{3}, \text { say. }
\end{aligned}
$$

4 G. H. Hardy, Divergent series, 1949, Theorem 65, p. 122.

5 Zygmund, Trigonometrical series, 1935, p. 55. 
Straightforward calculations give

$$
\Delta\left(\frac{r}{\log r}\right)=O\left(\frac{1}{\log r}\right)
$$

and

$$
\Delta^{2}\left(A_{n-r}^{\prime} \frac{r}{\log (r+1)}\right)=O\left(\frac{1}{\log r}\right) .
$$

Using (13) and (16) we have

$$
T_{2}=o(n),
$$

and by Lemma II we find

$$
T_{3}=o\left(\frac{n^{2}}{\log n}\right) .
$$

Now by (13) and (17) we have

$$
T_{1}=\sum_{r=1}^{n-2}(r+1) o(\log r) o\left(\frac{1}{\log r}\right)=o\left(n^{2}\right) .
$$

Thus

$$
\sum_{r=1}^{n} A_{n-r}^{\prime} r B_{r}(x) \frac{r}{\log (r+1)}=o\left(n^{2}\right)
$$

which completes the proof of Theorem 2.

Ravenshaw College 